\title{
TRELLIS-CODED 8-DPSK WITH POSTDETECTION COMBINING DIVERSITY FOR DIGTTAL LAND MOBILE RADIO
}

\author{
G. FEMENIAS and R. AGUSTI
}

Universitat Politècnica de Catalunya (UPC)

Apdo. 30.002, 08080 Barcelona (Spain)

\begin{abstract}
In this paper we analyze the performance of Rate $2 / 3$ postdetection combining diversity TCM-8DPSK systems. A simulated Rayleigh mobile radio channel and the presence of co-channel interferers have been assumed. Also, only the case where ideal interleaving/deinterleaving process is used to further combat the Rayleigh fading is considered. Bit error rate (BER) is obtained, through computer simulation, as a function of signal to noise ratio (SNR) and carrier to interference average power ratio (CIR). Results obtained for the QDPSK and TCM-8DPSK schemes have been considered as a reference to determine the performance improvements introduced by the coding diversity set-up.
\end{abstract}

\section{INTRODUCTION}

In recent years there has been increasing interest in digital signal transmission for UHF high capacity cellular mobile radio systems. Since the received signal is severely affected by the multipath propagation environment, some auxiliary techniques are necessary to reduce the fading effects. One of the most efficient and largely invoked techniques is space diversity reception, in which several signals received at different antennas are combined [1]. Furthermore, in a cellular mobile radio system the basis for reuse distance calculations is the minimum carrier to interference average power ratio (CIR) for which the radio link still offers acceptable quality. Then, a way of improving the capacity of the land mobile systen would consist of using digital modulation schemes with good CIR tolerances and without extra bandwidth requirements.

Trellis Coded Modulation (TCM), as introduced by Ungerboeck [2], has recently become a popular means of achieving coding gain on the AWGN channel and also on the Rayleigh fading channel, relative to an uncoded scheme with the same spectrum efficiency. Moreover, the gain is available without extra bandwidth requirements and with simple decoders utilizing the Viterbi algorithm. Our aim in this work is to investigate to what extent we can improve both the capacity and the performance of the cellular mobile radio system by using both a proper TCM scheme, suitable for Rayleigh fading environments, and a space diversity reception technique. In particular, in this paper we assess the BER-performance of the Rate $2 / 3$ postdetection equal-gain (EGC) and maximal-ratio (MRC) combining TCM-8DPSK modulation schemes, that is, combined coding/diversity techniques.

\section{SYSTEM MODEL}

The equivalent baseband transmission system is shown in Fig. 1. A non-coherent modulation has been adopted instead of a coherent one because of the difficulties in these systems to extract the carrier in a radio mobile environment. We have assumed the overall transfer function to be, in absence of channel distortion, a raised-cosine with a 0.5 roll-off parameter equally split into transmitter and receiver. In order to emphasize only the effects of the coding/diversity set-up, ideal interleaving process, perfect automatic frequency control (AFC) and perfect clock recovery are assumed.

At the emitter, a data input bit pair $s_{1}=\left(s_{0, j}, s_{1}, j\right)$ is fed into a rate $2 / 3$ trellis encoder at time $t=i T$, which generates the coded 3-bit word $m_{i}=\left(m_{0, j}, m_{1, i}, m_{2, j}\right)$ as a function of its state at the instant $t=(\mathrm{i}-1) \mathrm{T}$ and of the data input symbol $\mathrm{s}_{\mathrm{i}}$. The octal mapper, applying mapping by set partitioning [2], converts the 3-bit word $m_{i}$ into the coded 8-PSK symbol $a_{i}$ given by

$$
a_{1}=\exp \left\{j \cdot\left(m_{2 j} \cdot \pi+m_{1 j} \cdot \frac{\pi}{2}+m_{0,} \cdot \frac{\pi}{4}\right)\right\}
$$

These coded symbols are fed into the block interleaver that breaks the channel memory and makes full use of the error correction properties of the Ungerboeck code. Differential encoding of the interleaver output symbols, $b_{i}$, yields the coded 8-DPSK symbol

$$
c_{i}-c_{i-1} \cdot b_{i}
$$

which once filtered by the transmission filter, $h_{T}(t)$, provides the complex baseband transmitted signal,

$$
x(t)-\sum_{k} c_{k} \cdot h_{T}(t-k T)
$$




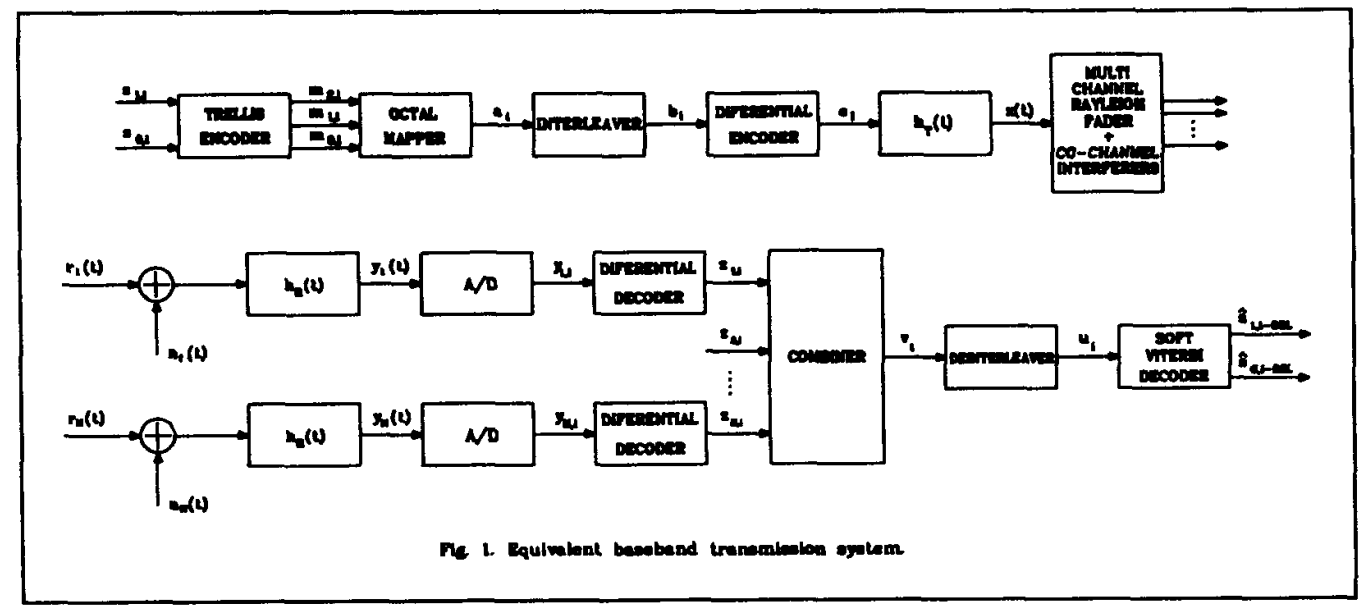

Assuming, in general, an M-branch diversity system, the $j-$ th $(j=1,2, \ldots, M)$ receiver input complex baseband signal can be expressed as

$$
r_{J}(t)=\sum_{k} c_{k} \cdot A_{0 J}(t) \cdot h_{T}(t-k T)+I_{j}(t)+w_{j}(t)
$$

where $w_{j}(t)$ is an additive complex white Gaussian noise and $I_{j}(t)$, assuming that $N$ is the total number of co-channel interferers, can be written as

$$
\begin{gathered}
I_{j}(t)-\sum_{m-1}^{N} i_{m \jmath}(t)- \\
-\sum_{m=1}^{N} \sum_{k} \alpha_{k, m} \cdot A_{m J}(t) \cdot h_{T}\left(t-k T-\tau_{m, J}\right)
\end{gathered}
$$

with $\left\{i_{m}(t)\right\}$ being co-channel interferer signals showing the same nature as the wanted one, $\left\{\alpha_{k, m}\right\}$ the data symbols transmitted by the $m$-th interferer and $\left(\tau_{\mathrm{mj}}\right\}$ the interferer signal delays with respect to the wanted signal. The signals $\left(A_{m j}(t)\right\}$ are uncorrelated complex-valued Gaussian stochastic processes which describe multiplicative non-frequency selective short-term fading. The received signal is filtered by the reception filter giving,

$$
\begin{gathered}
y_{j}(t)-\sum_{k} c_{k} \cdot A_{0, j}(t) \cdot h(t-k I)+ \\
+\sum_{m=1}^{N} \sum_{k} \alpha_{k, m} \cdot A_{m j}(t) \cdot h\left(t-k T-\tau_{m J}\right)+n_{j}(t)
\end{gathered}
$$

where $h(t)$ is the overall impulse response of the transmission channel in absence of Rayleigh distortion and $n_{j}(t)$ is an additive complex Gaussian noise. This signal is sampled by an $A / D$ converter at time $\mathrm{t}_{\mathrm{i}}=\mathrm{iT}-\tau$, where $-T / 2 \leq \tau \leq T / 2$ determines the sampling time, providing the complex sample,

$$
\begin{array}{r}
y_{j, 1}-c_{i} \cdot A_{0, j}\left(t_{i}\right) \cdot h(\tau)+\sum_{k+i} c_{k} \cdot A_{0, j}\left(t_{i}\right) \cdot h\left(t_{i}-k T\right)+ \\
+\sum_{m=1}^{N} \sum_{k} \alpha_{m, k} \cdot A_{m J}\left(t_{j}\right) \cdot h\left(t_{i}-k T-\tau_{m, j}\right)+n_{j}\left(t_{i}\right)
\end{array}
$$

This expression shows that the wanted sample is affected by a term of intersymbol interference, a term of co-channel interference and a term of Gaussian noise. Assuming a perfect clock recovery, $t=0$, we have $h(0)=1$ and $h((i-k) T)=0$, for all $k \neq i$, and so the ISI term vanishes. If we also assume, in order to stress the co-channel interference effects, that all the interferers are synchronized with our system, that is $\tau_{j}=0$, the complex sample at the output of the A/D converter will be

$$
\begin{gathered}
y_{j, 1}-c_{i} \cdot A_{0, j}\left(t_{i}\right)+\sum_{m-1}^{N} \alpha_{m, i} \cdot A_{m, j}\left(t_{i}\right)+n_{j}\left(t_{i}\right)- \\
-c_{i} \cdot A_{0, j}+\sum_{m-1}^{N} \alpha_{m, i} \cdot A_{m, j, 1}+n_{j, 1}
\end{gathered}
$$

For the Nyquist system one can assume the noise samples $\mathrm{n}_{\mathrm{j}, \mathrm{i}}$ to be uncorrelated and so, the average bit energy to spectral noise power density ratio is, for $\tau=0$,

$$
\frac{E_{b J}}{N_{0}}-\frac{E\left\{\left|A_{0, j, 1}\right|^{2}\right\} \cdot E\left\{\left|c_{i}\right|^{2}\right\}}{4 \cdot \sigma^{2}}
$$


where $E(\cdot)$ denotes expectation and $\sigma^{2}$ is the variance of the thermal noise in each dimension. The total carrier to interference average power ratio is given by

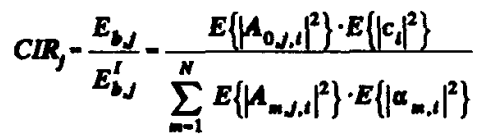

where $E_{b j}$ and $E_{b j}^{I}$ denote the average bit energy of the wanted and interferer signals, respectively, $j=1, \ldots, M$. In the following we will assume the normalization

$$
\begin{aligned}
& E\left\{\left|\boldsymbol{A}_{0, \mathrm{~J}, 1}\right|^{2}\right\}-E\left\{\left|c_{1}\right|^{2}\right\}-E\left\{\left|\alpha_{-, 1}\right|^{2}\right\}-1 \\
& E\left\{\left|A_{k, d, i}\right|^{2}\right\}-E\left\{\left|A_{n, \lambda, i}\right|^{2}\right\} ; k, n \in[1, N] \text {. }
\end{aligned}
$$

For Maximal Ratio Combining (MRC), symbol detection is achieved by a differential decoder which uses the received symbol at the instant $t=(i-1) T$ as phase reference [3]. For Equal Gain Combining (EGC), symbol detection is achieved by the same system but a limiter giving unity amplitude is used in the feed-forward signal path [3]. So, the detected symbol at the $j$-th receiver can be written as

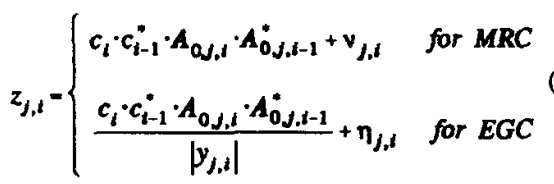

where $v_{j, i}$ and $\eta_{j, j}$ are complex noise samples which, due to the multiplication, are non-Gaussian and correlated. Once performed the differential detection, the output signals of the $M$ receivers are combined to give

$$
v_{i}=\sum_{j=1}^{M} z_{j, i}
$$

These samples (soft quantized symbols) are stored into the block deinterleaver in successive rows and read out in columns for soft Viterbi decoding.

The trellis encoder used at the transmitter side functions as a "finite state machine", then the Viterbi decoder will be optimum for estimating the maximum likelihood coded symbol sequence. But the noise samples at the Viterbi decoder input are non-Gaussian and correlated, for that, the use of a proper metric is needed. The cumulative metric that we have used for the path ending at state $\mu$ at time iT is given by the negative increase of the quadratic Euclidian distance over a symbo period [4],

$$
Q_{\mu, t}=-\sum_{k=0}^{L}\left|u_{t-k}-Q_{k, i-k-D E L}\right|^{2}
$$

where $\mathrm{L}$ and DEL represent the delays introduced by the soft Viterbi decoder and the interleaving/deinterleaving process, respectively, and $u_{i}$ is the deinterleaver matrix output symbol at the instant iT.

\section{RESULTS AND CONCLUSIONS}

Extensive computer simulations have been carried out in order to assess the BER-performance of Rate 2/3 two-branch postdetection combining diversity TCM-8DPSK modulation schemes with an 8 state Ungerboeck code [2], for a Rayleigh land mobile radio channel and in the presence of co-channel interference. Computer simulations have also been carried out for two-branch postdetection combining diversity QDPSK modulation scheme in order to provide a reference to determine the BER improvements introduced by the coding/diversity set-up. In this case, the detection process has been performed through a symbol by symbol hard decision algorithm. In all the programs we have assumed an ideal interleaving process, that is, the mobile radio channel appears as memoryless. Also, perfect automatic frequency control (AFC) and symbol synchronization are assumed.

In order to establish a comparison between different modulation strategies Fig. 2 shows the BER-performance of QDPSK, EGC TCM-8DPSK and MRC TCM-8DPSK systems with two-branch post-detection combining diversity. In particular, results comparing the $B E R$ versus $E_{\sqrt{ }} / N_{0}$ for different carrier to interference average power ratios (CIR) are presented. From these results we can extract the following conclusions. First of all, they point out that, for an infinite CIR and $E_{6} / N_{0}$ ratios greater than $6 \mathrm{~dB}$, the EGC TCM-8DPSK and MRC TCM-8DPSK systems perform better than the uncoded EGC QDPSK and MRC QDPSK systems. For example, at the BER of $10^{-3}$, of interest in digital speech transmission, the coded systems offer relative coding gains over the uncoded systems of about $5.5 \mathrm{~dB}$ and $5.9 \mathrm{~dB}$, respectively. Secondly, for an infinite $C I R$ and $E_{b} / N_{0}$ ratios lower than $6 \mathrm{~dB}$ the trellis coded modulation schemes are worse than uncoded systems and, thus, bit error rates in the area of $10^{-1}$ are not improved by TCM. Thirdly, for an infinite CIR, curves for EGC QDPSK, MRC QDPSK, EGC TCM-8DPSK and MRC TCM-8DPSK have asymptotic diversity orders near to $2.0,2.0,4.0$ and 4.6 , respectively. And, finally, in an interference limited land mobile radio system, remarkable improvement of the BER-performance can be attained for CIR greater than $10 \mathrm{~dB}$. In particular, for a BER of $10^{-3}$, MRC TCM-8DPSK, EGC TCM-8DPSK, MRC QDPSK and EGC QDPSK systems can cope with a CIR of $11.5 \mathrm{~dB}, 12.5 \mathrm{~dB}, 17 \mathrm{~dB}$ and $18 \mathrm{~dB}$, respectively.

Fig. 3 shows the results obtained for Rate $2 / 3$ TCM-8DPSK with and without postdetection combining diversity, for a Rayleigh land mobile radio channel and 


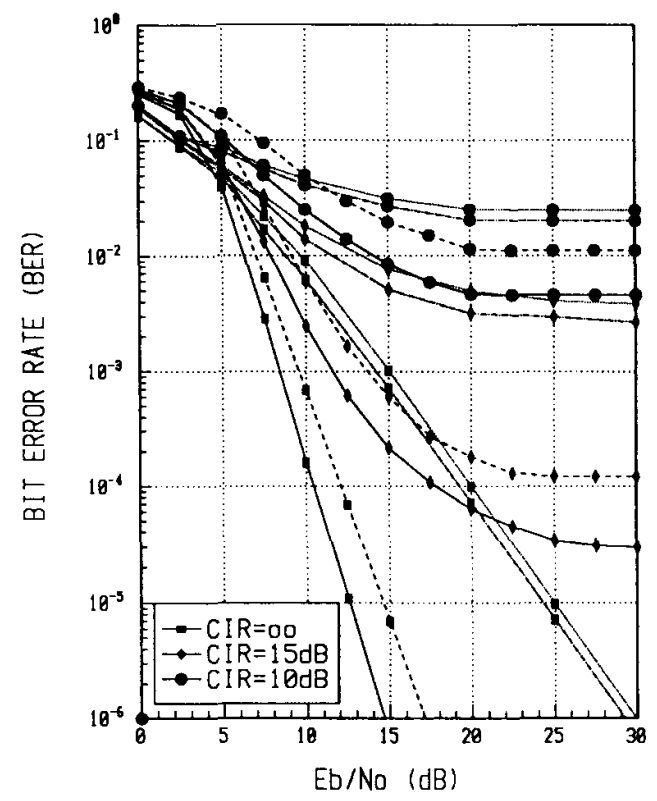

Fig. 2 BER-Performance of Postdetection EGC QDPSK $\quad$-........ NRC QDPSK $(\cdots-)$, KRC ICM-8DPSK (-) and E6C. TCN-8DPSK $(--)$ for a Rayleigh mobile channel and CIR as par ameter. $\quad(N=2, N=6)$

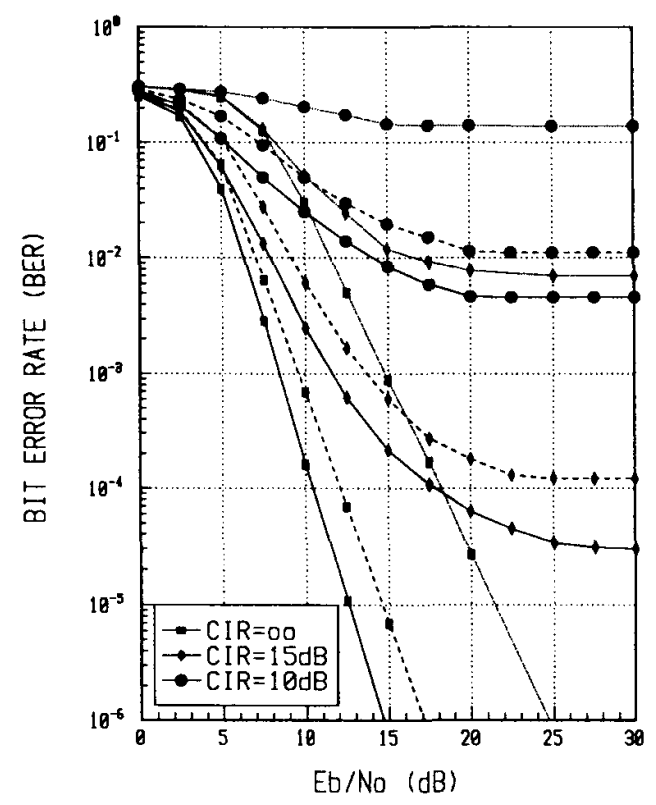

Fig. 3 BER-Performance of Rate $2 / 3$ TCM-8DPSK (…..), Postdetection EGC TCM-80PSK (----) and Postdetection MRC TCH-8OPSK $(-)$ for a Rayleigh channel and CIR as parameter. $(N=2, N=6)$

\section{REFERENCES}

CIR as parameter. Results for TCM-8DPSK without postdetection combining diversity were presented in [5].As we can see postdetection combining diversity is a powerful tool to further combat the effects of the Rayleigh fading. Roughly speaking, if TCM-8DPSK systems without diversity have an asymptotic behavior of order $n$, TCM-8DPSK systems with postdetection combining diversity have an asymptotic behavior of order $\mathrm{n}+1$. Also, at the BER of $10^{-3}$, the systems with postdetection EGC and MRC diversity offer a relative coding gain over the system without diversity near to $5.0 \mathrm{~dB}$ and $6.2 \mathrm{~dB}$, respectively. Furthermore, TCM systems with diversity are more robust than TCM systems whithout diversity in front of the effects of co-channel interference. In particular, in an interference limited system and for a BER of $10^{-3}$, MRC TCM-8DPSK, EGC TCM-8DPSK and TCM-8DPSK systems can withstand a CIR of $11.5 \mathrm{~dB}, 12.5 \mathrm{~dB}$ and $17.75 \mathrm{~dB}$, respectively.

\section{AGREEMENTS}

This work was supported by CICYT (Spain) under Grant TIC 880543.
[1] D.G. BRENNAN: Linear Diversity Techniques, Proc. IRE, vol. 47, 1959.

[2] G. UNGERBOECK: Channel Coding with MultilevellPhase Signals, IEEE Trans. on Inf. Theory, vol. IT-28, January 1982.

[3] F. ADACHI and J.D. PARSONS: Postdetection Diversity using a Modified Phase-Correction Loop for Digital Land Mobile Radio, IEE Proc., vol. 134, Pt. F, no. 1, February 1987.

[4] F. EDBAUER: Performance of Interleaved Trellis-Coded Differential 8-PSK Modulation over Fading Channels, IEEE Journal on Sel. Areas in Comm., vol 7, no. 9, December 1989.

[5] K. LEEUWIN, G. FEMENIAS and R. AGUST: Performance of Trellis-Coded 8-DPSK Modulation with Co-Channel Interference in Land Mobile Radio Channels, Conf. Rec. TENCON'89. 